Research Article

\title{
Reliability of Lower Extremity Muscle Power and Functional Performance in Healthy, Older Women
}

\author{
Konstantina Katsoulis $\mathbb{D}^{1},{ }^{1}$ Sunita Mathur $\mathbb{D}^{2}{ }^{2}$ and Catherine E. Amara $\mathbb{D}^{1}$ \\ ${ }^{1}$ University of Toronto, Faculty of Kinesiology and Physical Education, 55 Harbord Street, Toronto, Ontario M5S 2W6, Canada \\ ${ }^{2}$ University of Toronto, Department of Physical Therapy, Toronto, Canada \\ Correspondence should be addressed to Catherine E. Amara; cathy.amara@utoronto.ca
}

Received 25 July 2020; Revised 14 January 2021; Accepted 4 February 2021; Published 17 February 2021

Academic Editor: Carmela R. Balistreri

Copyright (C) 2021 Konstantina Katsoulis et al. This is an open access article distributed under the Creative Commons Attribution License, which permits unrestricted use, distribution, and reproduction in any medium, provided the original work is properly cited.

\begin{abstract}
Evaluation of the long-term reliability of muscle power and functional performance tests in older, healthy adults is warranted since determining whether performance is consistent over longer durations is more relevant for intervention studies. Objective. To assess the long-term test-retest reliability of measures of muscle power and lower body functional performance in healthy, nonexercising, older women. Methods. Data were derived from a nonexercising control group $(n=18$; age $=73.3(3.4)$ years; height $=159.6(7.7) \mathrm{cm}$; body mass $=69.5(12.7) \mathrm{kg}$; BMI $=27.3(4.8))$ of a randomized controlled trial of muscle power training in older women. Participants underwent lower extremity muscle power (Biodex) and functional testing (Short Physical Performance Battery, gait speed, 30-second chair stands, stair climbing, and 400-meter walk) at week 0 (baseline), 9, and 15. Results. For the upper leg, intraclass correlation coefficients (ICCs) were very high for knee extension power (0.90-0.97) and high to very high for knee flexion power (0.83-0.96). For lower-leg power, ICCs were high to very high for plantar flexion and dorsiflexion (0.83-0.96). ICCs for functional performance were moderate to very high (0.64-0.93). Coefficient of variation of the typical error $\left(\mathrm{CV}_{\mathrm{TE}}\right)$ was $<10.5 \%$ for knee extension/flexion power, $9.9-20.0 \%$ for plantar flexion/dorsiflexion power, and $1.9-14.9 \%$ for functional performance. Knee extension power and stair climb power demonstrated the highest reliability for muscle power and function, respectively. Mean values did not change over time, with the exception of the chair stands $(p<0.05)$; however, these changes were not considered clinically meaningful. Conclusions. The current study provides evidence supporting the long-term reliability of performance assessments of muscle power and lower body functional capacity over a period of up to 15 weeks in healthy, older women.
\end{abstract}

\section{Introduction}

Functional status is associated with hospitalization, health decline, and death in older adults [1-3]. Since muscle power (the product of force and velocity) is a critical predictor of functioning in older adults [4], the evaluation of physical function and muscle power is especially important in older women who are at a higher risk for age-related functional decline [5]. Reliability data for the more commonly used tests determining functional status in healthy, older adults is lacking. Analysis of video recordings, in other populations, for the assessment of motor skills performance has been shown to be reliable and comparable to live assessment [6] and could also be used to assess the reliability of functional performance in older adults. While studies typically report the short-term ( $\leq 2$ weeks) reliability of functional outcomes [7-9], the long-term reliability ( $>12$ weeks) [10] is needed to consider the potential impact of the typical age-related decline in functional performance in addition to measurement error. Buehring et al. investigated the short and longterm ( $\sim 3$ months) reliability of functional tests in older (70-95 years), community-dwelling adults [10]. While their data demonstrate stable results in men across all time points for jumping performance, gait speed, 5 chair stands (5 CS), Short Physical Performance Battery (SPPB), and grip strength, the women showed an apparent improvement in 5 CS performance and decreased performance in jump height and grip strength. Thus, while it is not clear whether jump 
height and grip strength were impacted by age-related declines, there did appear to be sex-related differences in the reliability of functional measures over the course of four months.

Since resistance training is one of the primary recommended treatments for attenuating age-related functional decline [11] and improving muscle power [12], investigating the reliability of measures over durations typically used for training studies ( 3 months [12]) is warranted. Therefore, the purpose of this study was to investigate the long-term (after 9 and 15 weeks) test-retest reliability of muscle power and functional performance tests in healthy, untrained, older women. A secondary purpose was to establish the inter- and intrarater reliability of multiple raters for functional performance using both in-person assessment and video recordings.

\section{Materials and Methods}

2.1. Participants. Data for this study come from the nonexercising control group of a randomized controlled trial (functional outcomes and power training in older women: F-POW; NCT02530723). Older women were recruited in the Greater Toronto Area through flyers, e-mail lists, and electronic newsletters. Inclusion criteria consisted of women, $\geq 65$ years old, untrained ( $<1$ hour/week of structured moderate/high intensity exercise and not currently participating in resistance training), and who provided medical clearance from their physician to participate in exercise. Exclusion criteria consisted of osteoporosis, diabetes, uncontrolled hypertension, or a new/unstable condition diagnosed in the last six months. The University of Toronto's Research Ethics Board approved the study (Protocol \#27773) which complies with the Declaration of Helsinki. The reliability analysis included 18 women (mean age $=73.3(3.4)$ years, mean height $=159.6(7.7) \mathrm{cm}$, mean body mass $=69.5(12.7) \mathrm{kg}$, and mean $\mathrm{BMI}=27.3(4.8)$ ).

2.2. Procedure. The Guidelines for Reporting Reliability and Agreement Studies [13] were followed. Sample size estimation was made using a web-based sample size calculator (https://wnarifin.github.io/ssc/ssicc.html) and methods from Walter et al. [14]. A previous reliability study testing muscle power in older adults [9] reported an intraclass correlation coefficient (ICC) of 0.96 . Using the minimum acceptable reliability of 0.75 [15], $\alpha=0.05,80 \%$ power, and two repetitions/raters per participant would require a sample size of at least 10 participants.

After obtaining consent, the Geriatric Depression Scale (15 questions), Mini-Mental State Examination, and RAND Physical Functioning Subscale were administered. Using a Jamar dynamometer, seated handgrip strength was measured with the elbow resting on the arm rail and at a 90degree angle. Participants were instructed to squeeze the dynamometer "as hard as possible" for five seconds. Three attempts with each hand were measured, and the peak value was recorded as hand grip strength (to the nearest $\mathrm{kg}$ ).
Testing was conducted at 3 time points: 1 (week 0), 2 (week 9), and 3 (week 15). Participants were asked to abstain from physical activity at least 24 hours prior to testing and to be well-hydrated. No other intervention or contact was administered to participants throughout the control period. The participants were asked to maintain their usual physical activity and dietary intake routines for the duration of the study. Only participants with complete functional data for at least 2/3 time points were included in the current analysis.

2.3. Body Composition. Body composition was assessed from whole-body scans using a Lunar Prodigy Dual-Energy X-Ray Absorptiometry Scanner (enCORE software, v. 6.50.069, 2002, General Electric Lunar Corporation, Madison, Wisconsin). Fat-free mass (kg) was calculated as the sum of total lean tissue plus bone mineral content. Body fat (\%) was calculated as total fat tissue/body mass $\times 100$. Body mass $(\mathrm{kg})$ was obtained using a standard scale.

2.4. Muscle Torque and Power. Muscle power was measured using a Biodex isokinetic dynamometer (System 4.0, Shirley, New York) with all testing completed by one rater. A warmup of usual pace walking within the lab for three to five minutes preceded the testing. The dominant leg was tested, and leg dominance was determined by asking "which leg would you kick a soccer ball with?" Participants were seated upright on the Biodex (hip angle 90 degrees) with straps across the chest, lap, and testing leg for stability. The dynamometer was aligned with the knee joint axis of rotation, and the cushioned pad of the lever-arm was placed approximately two inches above the lateral malleolus. Participants were instructed to perform all contractions "as hard and as fast as possible," and motivation was provided throughout the trial. Maximal voluntary isometric contraction (MVIC) of the knee extensors (KE) and knee flexors (KF) were measured at a knee angle of 90 degrees [9]. Each trial consisted of KE MVIC, and a brief rest followed by KF MVIC. Contraction time was five seconds. Three trials were performed with one minute rest between trials. The peak torque for each muscle group was recorded as MVIC (Newton-metres, Nm). After MVIC testing, 40\% of MVIC was calculated and used as the load intensity for testing muscle power using the isotonic mode of the Biodex. Ten isotonic contractions were performed combining both concentric KE and KF back-to-back (10 reps for each) using the participant's full range of motion. Two trials were completed $(2 \times 10$ repetitions $)$ and separated by a twominute rest. Subsequently, the same procedure was followed to test MVIC and power for plantar flexion (PF) and dorsiflexion (DF).

For PF and DF testing, the Biodex seat was reclined (hip angle 110 degrees). The right ankle was tested in all participants. The right leg was elevated such that the tibia was parallel to the floor (knee angle $\sim 145$ degrees). The right foot was strapped onto the foot plate, and the dynamometer axis of rotation was aligned with the lateral malleolus and support placed under the hamstrings. MVIC was tested in the neutral position (0 degrees), and $\mathrm{PF}$ and $\mathrm{DF}$ power were 
tested within a 30-degree range of motion (from 30 degrees of $\mathrm{PF}$ to 0 degrees). The torque $(\mathrm{Nm})$ and instantaneous velocity $(\mathrm{rad} / \mathrm{s})$, recorded using external software (Acqknowledge 4.1.1: Biopac Systems Inc., 2010), were multiplied to yield power (W) for each repetition. The highest of the 20 repetitions for each muscle group was used for analyses.

2.5. Functional Performance. Functional performance was tested using 6 tests: SPPB, 4-meter fast-pace walk (4 MFP), the 30 -second chair stands (30 sCS), stair climbing at usual (SCUP) and fast (SCFP) pace, and 400-meter walk (400 MW). A Sportline Tough Timer Stopwatch (E \& B Exercise, LLC, Yonkers, NY, USA) was used by raters for timing. The tests were video-recorded on an iPad mini for all three time points. Functional raters $(n=3)$ were experienced graduate students, trained by the PI. All functional raters were blinded to group allocation and the health history of the participants for the duration of the study. Functional performance was tested by a single rater at each testing session.

2.6. Inter-and Intrarater Reliability. The videos from the functional testing at time point 1 were used to assess interand intrarater reliability. To assess the interrater reliability, video recordings were viewed and scored to produce videorecorded scores by all three raters. The raters were unaware of the scores recorded by the other raters.

To assess intrarater reliability, the scores from the inperson (live) functional testing at time point 1 were compared with the video-recorded scores of the same rater. Intrarater reliability was compared for 2 raters only (rater 3 was omitted since this rater only tested 2 participants at time point 1). Rater 1 did not complete the $400 \mathrm{MW}$ video analyses. Therefore, intrarater $400 \mathrm{MW}$ data came from rater 2 only. The same stopwatch was used to score functional performance while viewing the video recordings.

2.7. Short Physical Performance Battery. The SPPB [16] consists of three components: (1) balance (feet together, semitandem, and tandem); (2) timed 4-meter walk at usual pace (4 MUP) starting with the toes at the start line and finishing once the first foot has completely crossed the 4meter line; (3) time to complete 5 CS as fast as possible, starting from sitting with feet flat on the floor and arms crossed on the shoulders. Time was started upon rising from the chair and ended after the 5th chair stand. A standard firm chair without handrails with a height of 45 inches was used for all chair testings. The faster of two trials (separated by $\sim 30$-second rest) for the $4 \mathrm{MUP}$ and $5 \mathrm{CS}$ were rounded to the nearest 0.1 seconds and were used for analysis. The score $(0-4)$ for the three components was summed for the SPPB total score $(0-12)$.

2.8. Four-Meter Fast Pace Walk. For the 4 MFP [17], participants were asked to stand approximately three meters behind the start line and walk at a maximal pace until they were approximately three meters passed the finish line. Timing (to the nearest 0.1 seconds) started when the foot crossed the starting line and stopped when the foot crossed the finish line.

2.9. Thirty-Second Chair Stands. Starting from sitting with feet flat on the floor and arms crossed at the wrists on the shoulders, participants were instructed to perform as many full chair stands as possible in 30 seconds [18]. Only one trial was performed. Timing started upon rising from the chair and chair stand count ended at 30 seconds. If the participant was at least halfway to completing the last chair stand at the 30 -second mark, that chair stand was counted in the total number recorded.

2.10. Stair Climbing. A 13-step staircase with handrails in a well-lit area was used for stair ascent. Step height averaged 18 centimeters and step width averaged 28 centimeters. The total vertical height of the steps was 2.34 meters. Participants were asked to stand at the base of the stairs with feet together and to grab the handrail if necessary during ascent. Upon the instruction "ready, set, go," timing (to the nearest $0.1 \mathrm{sec}$ onds) started when the first foot left the ground and stopped when both feet were placed at the top of the 13th step. Stair ascent was measured during a "usual" pace (SCUP) and during a "fast" pace (SCFP) with instructions to ascend "as quickly and as safely as possible." The faster of two trials for each test was chosen for analysis. Stair climb power (SCP) for SCFP was calculated as SCP $(\mathrm{W})=($ (body mass in $\mathrm{kg}) \times\left(9.8 \mathrm{~m} / \mathrm{s}^{2}\right) \times($ stair height in meter $\left.)\right) /($ time in seconds $)$ [19].

2.11. Four-Hundred-Meter Walk. The $400 \mathrm{MW}$ test [20] consisted of 10 laps around cones placed 20 meters apart in a corridor. Participants were instructed to walk "as quickly and as safely as possible." After a two-minute walking warmup, time to complete 10 laps was recorded (to the nearest 0.1 seconds). Timing began when the participant lifted their foot off the ground at the starting line and ended when the first foot crossed the line at the end of the 10th lap. Standard encouragement was provided.

2.12. Statistical Analysis. Means and SD were calculated. Normality of data was tested with the Shapiro-Wilk test [21] and was visually inspected. The repeated measures analysis of variance (for normally distributed data) and Friedman test (for nonnormally distributed data) were used to test for differences in the means across time points. Intraclass correlation coefficients (ICC) using two-way mixed effects with absolute agreement were calculated for inter/intrarater reliability and time points 1 and 2;2 and 3; 1 and 3. Thresholds to describe ICCs were very low $=<0.20$, low $=0.20-0.50$, moderate $=0.50-0.75, \quad$ high $=0.75-0.90$, very high $=0.90-0.99$, and extremely high $=>0.99$ [15]. Statistical analyses were performed using IBM SPSS (v. 24, New York, USA). Coefficient of variation of the typical error $\left(\mathrm{CV}_{\mathrm{TE}}\right)$ [22] was calculated with Microsoft Excel (v. 15.0.5233.1000, Washington, USA) using the standard deviation of the change score/average means of both time points $\times 100$ for time points 
1 and 2, 2 and 3, and 1 and 3. Effect sizes were calculated using means and SD of change scores for post-hoc matched pairs statistical tests using G*Power Statistical Software [23] for time points 1 and 2, and 1 and 3. Bland-Altman plots were created using SigmaPlot (version 14, Illinois, USA) with time points 1 and 3 only (for brevity). Simple linear regression was performed using SigmaPlot with the difference score (time points 3-1) as the dependent variable and the mean score (time point $(1+3) / 2$ ) as the independent variable for all measures to test for bias in these variables.

\section{Results}

Twenty-one participants were randomized to the control group. Three participants discontinued participation after baseline testing (two did not like the group allocation and one indicated a lack of time). Thus, a total of 18 participants were included in the reliability study. For the 18 participants included, two participants dropped out after the midpoint testing (reasons included a lack of time and change in health status) and one participant completed the third functional testing session but did not return for the dynamometer testing (due to travel). Dynamometer data are missing for the 2 nd time point for one participant (due to travel), power data are missing for one participant at time point 3 (technical issues), and stair climb data are missing for one participant at time point 1 (technical issues). Thus, the number of participants that completed all three testing time points was $16 / 18$ for functional testing and 13/18 for muscle power. Baseline participant characteristics are listed in Table 1. Overall, the participants were high-functioning (median RAND physical functioning score $=85$ and median MiniMental State Examination score $=30$ ) and at low risk for depression (median Geriatric Depression Scale score $=0$ ). Mean blood pressure was normal (120/78 $\mathrm{mm} \mathrm{Hg})$, and most participants were taking only one medication. Means (SD) for all participants that were included $(n=18)$, regardless of missing data, are available online in Tables S1 (torque and power) and S2 (functional performance). The complete data (means and SD) that were used in statistical testing are listed in Table 2 (torque, $n=14$; power, $n=13$; physical function, $n=16)$.

3.1. Inter- and Intrarater Reliability. Interrater reliability data are reported in Table 3 and indicated very high to extremely high [15] reliability with ICCs ranging from 0.95 to $1.00(p<0.05)$. Intrarater reliability (Table 4$)$ demonstrated high to very high ICCs ranging from 0.85 to 1.00 $(p<0.05)$.

3.2. Torque and Power. There were no significant differences in means for torque and power across time points $(p \geq 0.05$; Table 2). For torque and power of the knee extensors and flexors, $\mathrm{CV}_{\mathrm{TE}}$ between all time points ranged from $5.7 \%$ to $10.5 \%$. $\mathrm{CV}_{\mathrm{TE}}$ for the plantar flexors and dorsiflexors ranged from 9.7 to $20.0 \%$ (Table 5 ). ICCs for all measures of torque and power $(p<0.01)$ were $\geq 0.90$ indicating very high reliability, with the exception of dorsiflexion power from time points 2 and 3 and 1 and 3 (ICCs $=0.83$ and 0.88 , respectively; Table 5) where reliability was considered high. BlandAltman plots can been found in Figures S1-S5 online. The majority of the data fell within the limits of agreement. In addition, there were no significant regression models (all $p \geq 0.05$, data not shown), suggesting no bias in the data.

3.3. Functional Performance. For balance, almost all participants received maximal scores (4/4) at all time points (data not shown). There were no significant differences in the means of the functional performance tests across time points ( $p \geq 0.05$; Table 2) except for the chair stands (5 CS and $30 \mathrm{sCS}$ ) which improved significantly from time point 1 to $3(p<0.05) . \mathrm{CV}_{\mathrm{TE}}$ for functional performance ranged from 1.9 to $14.9 \%$, and ICCs $(p<0.01)$ ranged from 0.64 to 0.95 reflecting moderate to very high reliability (Table 6). Stair climb power demonstrated the highest reliability, with ICCs of 0.93-0.95 for all time point comparisons. BlandAltman plots are shown in Figures S6-S14. Regression models for the $30 \mathrm{sCS}\left[R^{2}=0.29, F(1,15)=5.80, p=0.03\right]$ and the $400 \mathrm{MW}\left[R^{2}=0.29, F(1,15)=5.82, p=0.03\right]$ were significant.

\section{Discussion}

The main findings include the following: (1) isotonic muscle power demonstrated high to very high reliability over a nineand fifteen-week duration with knee extension power being the most stable measure; (2) all of the functional tests studied demonstrated reliability that ranged from moderate to high, with stair climb power exhibiting the highest reliability over durations that are commonly used in training studies for older adults [12]; (3) standardized testing protocols, despite having multiple raters and minimal participant familiarization, yielded consistent performance measurements.

The current study utilized the isotonic mode of the Biodex dynamometer to evaluate muscle power and hence used a less common velocity-dependent power measure where movement velocity is measured and the load used can be quantified [25]. The current results for KE power are similar with a previous investigation in older men and women investigating the one-week test-retest reliability of KE power using the isotonic mode [9]. The ICC reported in that study using a similar intensity ( $50 \%$ of MVC) of muscle power was $0.96(\mathrm{CI}=0.94-0.98)$, comparing well with the current study's ICCs (0.96-0.97 for all time point comparisons). Thus, KE power appears to be well maintained in untrained, healthy older women over a duration of 15 weeks. For PF and DF power, the ICCs ranged from 0.83 to 0.96 , indicating high to very high reliability. These findings are in agreement with Webber et al. who investigated the reliability of lower-leg muscle performance testing in older women using the isotonic mode of a dynamometer over a one-week test-retest interval [22]. They tested $\mathrm{PF}$ and $\mathrm{DF}$ power at $50 \%$ of MVC, and reported ICCs of $0.92(\mathrm{CI}=0.68-0.93)$ for $\mathrm{PF}$ power and $0.95(\mathrm{CI}=0.90-0.98)$ for $\mathrm{DF}$ power, with a $\mathrm{CV}_{\mathrm{TE}}$ of 14 and 12\%, respectively. The current study did not determine one-week test-retest results; however, in the 
TABLE 1: Baseline characteristics of participants $(n=18)$.

\begin{tabular}{|c|c|c|c|}
\hline & Mean (SD) & Median & (Minimum, maximum) \\
\hline Age (y) & $73.3(3.4)$ & 74.3 & $(66.9,79.5)$ \\
\hline Height $(\mathrm{cm})$ & $159.6(7.7)$ & 161.0 & $(142,170)$ \\
\hline Body mass $(\mathrm{kg})$ & $69.5(12.7)$ & 69.0 & $(48.4,96.2)$ \\
\hline BMI $\left(\mathrm{kg} / \mathrm{m}^{2}\right)$ & $27.3(4.8)$ & 26.6 & $(19.9,38.2)$ \\
\hline Body fat $(\%)$ & $40.0(8.2)$ & 41.0 & $(28.0,56.5)$ \\
\hline Fat-free mass (kg) & $39.8(7.4)$ & 40.5 & $(18.7,52.4)$ \\
\hline Hand grip strength $(\mathrm{kg})$ & $26.5(5.4)$ & 27.0 & $(16.0,36.0)$ \\
\hline Systolic BP (mm Hg) & $119.5(10.4)$ & 120.0 & $(95,140)$ \\
\hline Diastolic BP (mm Hg) & $77.9(7.7)$ & 77.5 & $(65,90)$ \\
\hline RAND Physical Functioning Subscale & & 85 & $(40,100)$ \\
\hline Geriatric Depression Scale & & 0 & $(0,5)$ \\
\hline Mini-Mental State Examination & & 30 & $(29,30)$ \\
\hline Medications (\#) & & 1 & $(0,10)$ \\
\hline
\end{tabular}

${ }^{a}$ Data are means (SD), except for the RAND Physical Functioning Subscale, Geriatric Depression Scale, Mini-Mental State Examination, and medications, where median $(\min , \max )$ are reported since data were not normally distributed; $\mathrm{BMI}=$ body mass index, $\mathrm{PF}=$ physical functioning, $\mathrm{BP}=$ blood pressure.

TABLE 2: Muscle torque ( $n=14)$, power $(n=13)$, and functional performance $(n=16)$ for time points 1 (week 0$), 2$ (week 9 ), and 3 (week 15$)$.

\begin{tabular}{|c|c|c|c|c|c|c|}
\hline & \multicolumn{3}{|c|}{ Time point } & \multirow{2}{*}{$p$ value } & \multirow{2}{*}{$\begin{array}{c}\text { ES } \\
1 \text { and } 2\end{array}$} & \multirow{2}{*}{$\begin{array}{c}\text { ES } \\
1 \text { and } 3\end{array}$} \\
\hline & 1 & 2 & 3 & & & \\
\hline KET & $92(35)$ & $92(26)$ & $92(28)$ & 0.98 & 0.05 & 0.02 \\
\hline KEP & $158(43)$ & $162(39)$ & $164(38)$ & 0.29 & 0.37 & 0.38 \\
\hline KFP & $125(31)$ & $128(36)$ & $132(32)$ & 0.37 & 0.20 & 0.33 \\
\hline PFP & $109(36)$ & $104(37)$ & $112(40)$ & 0.35 & 0.32 & 0.16 \\
\hline DFP & $12(4)$ & $12(5)$ & $11(3)$ & 0.47 & 0.12 & 0.26 \\
\hline SPPB & $12(9,12)$ & $12(9,12)$ & $12(9,12)$ & 0.06 & 0.20 & 0.41 \\
\hline 4 MUP (s) & $3.4(0.5)$ & $3.2(0.5)$ & $3.3(0.5)$ & 0.40 & 0.27 & 0.30 \\
\hline $4 \mathrm{MFP}(\mathrm{s})$ & $2.1(0.3)$ & $2.2(0.3)$ & $2.1(0.3)$ & 0.55 & 0.15 & 0.09 \\
\hline $400 \mathrm{MW}$ (s) & $279.2(26.5)$ & $271.8(15.3)$ & $268.3(16.0)$ & 0.09 & 0.37 & 0.50 \\
\hline $5 \mathrm{CS}(\mathrm{s})$ & $10.5(2.8)$ & $9.7(1.6)$ & $9.1(2.2)^{*}$ & 0.02 & 0.36 & 0.76 \\
\hline 30 sCS (reps) & $14(3)$ & $15(3)$ & $16(5)^{*}$ & 0.01 & 0.18 & 0.72 \\
\hline $\operatorname{SCUP}(s)(n=15)$ & $7.9(1.2)$ & $7.7(1.3)$ & $7.6(1.3)$ & 0.44 & 0.19 & 0.35 \\
\hline $\operatorname{SCFP}(s)(n=15)$ & $6.0(1.1)$ & $6.0(0.9)$ & $6.0(1.0)$ & 0.98 & 0.03 & 0.05 \\
\hline $\operatorname{SCP}(\mathrm{W})(n=14)$ & $273.3(76.7)$ & $263.2(57.0)$ & $271.2(67.9)$ & 0.51 & 0.26 & 0.06 \\
\hline
\end{tabular}

${ }^{a}$ Data are mean (SD), except for the SPPB, where medians (min, max) are displayed since data were not normally distributed; repeated measures ANOVA used to test means across time points, except for the SPPB which was tested using Friedman test; ES = Cohen's $d$ effect size for time points 1 and 2 and 1 and 3 , except for the SPPB which was calculated using $z / \sqrt{ } N$ for nonparametric data [24]. KET: isometric knee extension torque; KEP: knee extension power; KFP: knee flexion power; PFP: plantar flexion power; DFP: dorsiflexion power; SPPB: Short Physical Performance Battery; 4 MUP: 4-meter usual pace walk; 4 MFP: 4-meter fast pace walk; $400 \mathrm{MW}$ : 400-meter walk; 5 CS: 5 chair stands; 30 sCS: 30-second chair stands; SCUP: stair climb usual pace; SCFP: stair climb fast pace; SCP: stair climb power for the SCFP; ${ }^{*} p<0.05$. For the $5 \mathrm{CS}$ and $30 \mathrm{sCS}$, time point 3 was significantly different from time point 1.

TABLE 3: Interrater reliability of functional performance testing for three raters for video-recorded scores for time point 1 ( $n=17$ ).

\begin{tabular}{lccc}
\hline Test & ICC & $95 \%$ CI & $p$ value \\
\hline 4 MUP & 0.95 & $0.87-0.98$ & $<0.001$ \\
4 MFP & 0.98 & $0.94-0.99$ & $<0.001$ \\
5 CS & 0.97 & $0.72-0.99$ & $<0.001$ \\
30 sCS & 1.00 & $0.99-1.00$ & $<0.001$ \\
SCUP & 0.97 & $0.92-0.99$ & $<0.001$ \\
SCFP & 0.99 & $0.97-1.00$ & $<0.001$ \\
400 MW & 1.00 & $1.00-1.00$ & $<0.001$ \\
$(n=15)$ & & & \\
\hline
\end{tabular}

${ }^{2}$ ICC: intraclass correlation coefficient; CI: 95\% confidence intervals; 4 MUP: 4-meter usual pace walk; 4 MFP: 4-meter fast pace walk; 5 CS: 5 chair stands; 30 sCS: 30 -second chair stands; SCUP: stair climb usual pace; SCFP: stair climb fast pace; $400 \mathrm{MW}: 400$-meter walk. All ICCs were significant; ${ }^{*} p<0.05$. 
TABLE 4: Intrarater reliability of functional performance testing between video-recorded scores and live data collection for two raters for time point 1 ; rater 1 (R1): $n=9$ participants; rater 2 (R2): $n=6$ participants.

\begin{tabular}{|c|c|c|c|c|}
\hline Test & Rater & ICC & $95 \% \mathrm{CI}$ & $p$ value \\
\hline \multirow{2}{*}{4 MUP } & $\mathrm{R} 1$ & 0.85 & $-0.13-0.97$ & $<0.001$ \\
\hline & $\mathrm{R} 2$ & 0.85 & $-0.01-0.98$ & 0.01 \\
\hline \multirow{2}{*}{$4 \mathrm{MFP}$} & $\mathrm{R} 1$ & 0.92 & $0.65-0.98$ & $<0.01$ \\
\hline & $\mathrm{R} 2$ & 0.89 & $0.24-0.99$ & 0.02 \\
\hline \multirow{2}{*}{$5 \mathrm{CS}$} & $\mathrm{R} 1$ & 0.99 & $0.80-1.00$ & $<0.001$ \\
\hline & $\mathrm{R} 2$ & 1.00 & $0.97-1.00$ & $<0.001$ \\
\hline \multirow{2}{*}{$30 \mathrm{sCS}$} & $\mathrm{R} 1$ & 0.99 & $0.95-1.00$ & $<0.001$ \\
\hline & $\mathrm{R} 2$ & 1.00 & $0.98-1.00$ & $<0.001$ \\
\hline \multirow{2}{*}{ SCUP } & $\mathrm{R} 1$ & 0.94 & $0.68-0.99$ & $<0.001$ \\
\hline & $\mathrm{R} 2$ & 0.95 & $0.69-0.99$ & $<0.01$ \\
\hline \multirow{2}{*}{ SCFP } & $\mathrm{R} 1$ & 0.98 & $0.93-1.00$ & $<0.001$ \\
\hline & $\mathrm{R} 2$ & 0.98 & $0.50-1.00$ & $<0.001$ \\
\hline $\begin{array}{l}400 \mathrm{MW} \\
(n=4)\end{array}$ & $\mathrm{R} 2$ & 1.00 & $1.00-1.00$ & $<0.001$ \\
\hline
\end{tabular}

${ }^{a}$ ICC: intraclass correlation coefficient; CI: 95\% confidence intervals; 4 MUP: 4-meter usual pace walk; 4 MFP: 4 -meter fast pace walk; 5 CS: 5 chair stands; 30 sCS: 30-second chair stands; SCUP: stair climb usual pace; SCFP: stair climb fast pace; $400 \mathrm{MW}$ : 400-meter walk. All ICCs were significant; ${ }^{*} p<0.05$.

TABle 5: Reliability data for muscle torque $(\mathrm{Nm})$ and muscle power $(\mathrm{W})$ for time points 1 and 2 (weeks $0-9 ; n=17$ ), 2 and 3 (weeks 9-15; $n=13$ ), and 1 and 3 (weeks $0-15 ; n=14$ ).

\begin{tabular}{|c|c|c|c|c|c|c|}
\hline & \multicolumn{3}{|c|}{$\mathrm{CV}_{\mathrm{TE}}$} & \multicolumn{3}{|c|}{$\begin{array}{l}\text { ICC }(\mathrm{CI}) \\
p \text { value }\end{array}$} \\
\hline & 1 and 2 & 2 and 3 & 1 and 3 & 1 and 2 & 2 and 3 & 1 and 3 \\
\hline KET (Nm) & 9.5 & $\begin{array}{c}8.0 \\
(n=14)\end{array}$ & $\begin{array}{c}10.1 \\
(n=15)\end{array}$ & $\begin{array}{c}0.96(0.89-0.99) \\
<0.001\end{array}$ & $\begin{aligned} 0.97 & (0.89-0.99) \\
& <0.001\end{aligned}$ & $\begin{aligned} 0.96 & (0.87-0.99) \\
& <0.001\end{aligned}$ \\
\hline KEP (W) & 5.7 & 6.1 & 6.6 & 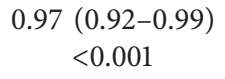 & $\begin{aligned} 0.97 & (0.90-0.99) \\
& <0.001\end{aligned}$ & $\begin{array}{c}0.96(0.88-0.99) \\
<0.001\end{array}$ \\
\hline $\mathrm{KFP}(\mathrm{W})$ & 9.7 & 6.7 & 10.5 & $\begin{array}{c}0.93(0.80-0.97) \\
<0.001\end{array}$ & $\begin{aligned} 0.97 & (0.90-0.99) \\
& <0.001\end{aligned}$ & $\begin{aligned} 0.90 & (0.68-0.97) \\
& <0.001\end{aligned}$ \\
\hline PFP (W) & 9.9 & 14.0 & 15.2 & $\begin{array}{c}0.96(0.89-0.99) \\
<0.001\end{array}$ & $\begin{aligned} 0.92 & (0.74-0.98) \\
& <0.001\end{aligned}$ & $\begin{aligned} 0.91 & (0.72-0.97) \\
& <0.001\end{aligned}$ \\
\hline DFP (W) & 15.9 & 20.0 & 14.8 & 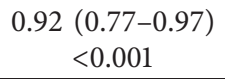 & $\begin{array}{c}0.83(0.47-0.95) \\
0.002\end{array}$ & $\begin{aligned} 0.88 & (0.62-0.96) \\
& <0.001\end{aligned}$ \\
\hline
\end{tabular}

${ }^{\mathrm{a}} \mathrm{CV}_{\mathrm{TE}}$ : coefficient of variation of the typical error (\%); ICC: intraclass correlation coefficient; CI: $95 \%$ confidence interval; KET: isometric knee extension torque; KEP: knee extension power; KFP: knee flexion power; PFP: plantar flexion power; DFP: dorsiflexion power. All ICCs were significant; ${ }^{*} p<0.05$.

shorter test-retest period (nine weeks), PF power ICC was $0.96\left(\mathrm{CI}=0.89-0.99, \mathrm{CV}_{\mathrm{TE}}=9.9 \%\right)$, and DF power ICC was $0.92\left(\mathrm{CI}=0.77-0.97, \mathrm{CV}_{\mathrm{TE}}=15.2 \%\right)$. Therefore, the very high test-retest reliability of lower-leg isotonic power previously demonstrated over one week is maintained over nine weeks in a healthy older population.

The current study's ICCs for functional performance testing ranged from 0.64 to 0.95 , indicating moderate to very high reliability, and all $\mathrm{CV}_{\mathrm{TE}}$ were $<15 \%$ over 15 weeks. In a 2013 systematic review of the properties of physical performance measures in community-dwelling older adults, the SPPB was recommended for use since it was found to have good intrarater reliability (ICCs $>0.7$ for total score, 4 MUP, and 5CS) and validity [26]. Mijnarends et al. reported several short-term reliability statistics for several functional tests, including but not limited to walking tests varying in length from two meters to one kilometer $(\mathrm{ICC}=0.94)$, the 6 minute walk test (ICCs $=0.88-0.94)$, and the $30 \mathrm{sCS}$
(0.84-0.92), which all reflect high to very high consistency. The current study also demonstrates high to very high reliability in most functional performance tests, with some tests (e.g., walking tests) demonstrating moderate reliability over 15 weeks (Table 6). Although these ICCs were moderate, the low $\mathrm{CV}_{\mathrm{TE}}(<9.6 \%$, Table 6$)$ and consistency in the means $(p>0.05$, Table 2) support their use for long-term reliability. Only one study from the review by Mijnarends et al. investigated the long-term reliability of the SPPB [27] (participants were moderately to severely disabled women) and reported average ICCs for SPPB score that declined gradually over time from six months $(\mathrm{ICC}=0.77)$ to 36 months $(\mathrm{ICC}=0.51)$. Their six-month average ICC for SPPB score is slightly lower compared with the current study's average ICC $(0.87$, range $=0.82-0.90)$. Thus, while a number of studies have reported strong short ( 1-2 week) test-retest reliability of functional performance in older clinical populations with ICCs ranging from 0.76 to $0.95[19,27,28]$, the 
TABLE 6: Reliability data for functional performance for time points 1 and 2 (weeks $0-9, n=18$ ), 2 and 3 (weeks 9-15, $n=16$ ), and 1 and 3 (weeks $0-15, n=16$ ).

\begin{tabular}{|c|c|c|c|c|c|c|}
\hline & \multicolumn{3}{|c|}{$\mathrm{CV}_{\mathrm{TE}}$} & \multicolumn{3}{|c|}{$\begin{array}{l}\text { ICC }(\mathrm{CI}) \\
p \text { value }\end{array}$} \\
\hline & 1 and 2 & 2 and 3 & 1 and 3 & 1 and 2 & 2 and 3 & 1 and 3 \\
\hline SPPB & 3.6 & 3.5 & 3.8 & $\begin{array}{c}0.90(0.73-0.96) \\
<0.001\end{array}$ & $\begin{array}{cl}0.88 & (0.65-0.96) \\
& <0.001\end{array}$ & $\begin{aligned} 0.82 & (0.44-0.94) \\
& <0.001\end{aligned}$ \\
\hline 4 MUP (s) & 10.1 & 8.7 & 9.6 & $\begin{array}{c}0.76(0.36-0.91) \\
<0.01\end{array}$ & $\begin{array}{c}0.81(0.45-0.93) \\
<0.01\end{array}$ & $\begin{array}{c}0.74(0.29-0.91) \\
<0.01\end{array}$ \\
\hline $4 \mathrm{MFP}(\mathrm{s})$ & 10.3 & 5.7 & 9.1 & $\begin{array}{c}0.77(0.40-0.91) \\
<0.01\end{array}$ & $\begin{array}{cl}0.91 & (0.73-0.97) \\
& <0.001\end{array}$ & $\begin{array}{c}0.69(0.10-0.89) \\
0.02\end{array}$ \\
\hline $400 \mathrm{MW}$ (s) & 5.0 & 1.9 & 5.6 & $\begin{aligned} 0.87 & (0.66-0.95) \\
& <0.001\end{aligned}$ & $\begin{aligned} 0.93 & (0.80-0.98) \\
& <0.001\end{aligned}$ & $\begin{array}{c}0.64(0.05-0.87) \\
0.02\end{array}$ \\
\hline $5 \mathrm{CS}(\mathrm{s})$ & 14.9 & 10.3 & 12.7 & $\begin{array}{c}0.71(0.25-0.89) \\
0.01\end{array}$ & $\begin{array}{c}0.84(0.57-0.95) \\
<0.001\end{array}$ & $\begin{aligned} 0.81 & (0.30-0.94) \\
& <0.001\end{aligned}$ \\
\hline 30 sCS (reps) & 9.2 & 11.8 & 10.6 & $\begin{aligned} 0.90 & (0.75-0.96) \\
& <0.001\end{aligned}$ & $\begin{aligned} 0.85 & (0.57-0.95) \\
& <0.001\end{aligned}$ & $\begin{aligned} 0.88 & (0.52-0.96) \\
& <0.001\end{aligned}$ \\
\hline SCUP (s) & $9.6(n=17)$ & 6.8 & $7.8(n=15)$ & $\begin{aligned} 0.88 & (0.65-0.96) \\
& <0.001\end{aligned}$ & $\begin{aligned} 0.91 & (0.73-0.97) \\
& <0.001\end{aligned}$ & $\begin{aligned} 0.86 & (0.60-0.95) \\
& <0.001\end{aligned}$ \\
\hline SCFP (s) & $7.9(n=17)$ & 8.6 & $9.5(n=15)$ & $\begin{aligned} 0.93 & (0.82-0.98) \\
& <0.001\end{aligned}$ & $\begin{array}{c}0.80(0.42-0.93) \\
<0.01\end{array}$ & $\begin{aligned} 0.82 & (0.45-0.94) \\
& <0.01\end{aligned}$ \\
\hline SCP (W) & $9.8(n=16)$ & $6.9(n=15)$ & $8.9(n=15)$ & $\begin{aligned} 0.93 & (0.80-0.98) \\
& <0.001\end{aligned}$ & $\begin{aligned} 0.95 & (0.86-0.98) \\
& <0.001\end{aligned}$ & $\begin{aligned} 0.94 & (0.82-0.98) \\
& <0.001\end{aligned}$ \\
\hline
\end{tabular}

${ }^{\mathrm{a}} \mathrm{CV}_{\mathrm{TE}}$ c coefficient of variation of the typical error (\%); ICC: intraclass correlation coefficient; CI: 95\% confidence interval; SPPB: Short Physical Performance Battery; 4 MUP: 4-meter usual pace walk; 4MFP: 4-meter fast pace walk; 400 MW: 400 -meter walk; 5 CS: 5 chair stands; 30 sCS: 30 -second chair stands; SCUP: stair climb usual pace; SCFP: stair climb fast pace; SCP: stair climb power for the SCFP; all ICCs were significant; ${ }^{*} p<0.05$.

reliability of functional performance measures appears to be compromised over longer durations in older adults with physical limitations. While it might be expected that healthy older adults will exhibit more stable functional performance over time compared with a clinical population, the welldocumented age-related declines in muscle mass [29], strength, and power [30] call into question over what period of time functional performance remains stable in this population. The present study provides evidence that a variety of functional tests which are commonly used in older adults can remain relatively stable over $\sim 4$ months. However, studies investigating the reliability of these tests over longer durations ( $\geq 6$ months) are needed.

The means for power and functional performance across time points did not change significantly, with the exception of the chair stand tests. There were small but significant changes that occurred from time points 1 and 3 for the 5 CS (faster time: -1.4 seconds) and the $30 \mathrm{sCS}$ (increase in chair stands: 1.6 reps). These average changes are less than the estimated minimally important changes found in the literature for clinical populations, which were 1.7 seconds for the 5 CS [31] and 3.3 chair stands for the $30 \mathrm{sCS}$ [32]. This suggests that although a learning effect might have occurred which resulted in improved chair stand performance, these changes were not clinically meaningful. In agreement with these findings, another investigation found that chair stand performance improved significantly in older women over $\sim 4$ months; however, it remained stable in older men [10]. Chair stand tests might represent a more ambiguous performance test where more practice is needed initially to decrease small "practice effects," at least in older women. Data from the Lifestyle Interventions and Independence for Elders Pilot
Study [33] reported meaningful changes of 0.4-1.5 units for the SPPB, 0.08 meters/second for the 4MUP, and 50-60 seconds for the $400 \mathrm{MW}$. Using those estimates, the mean changes in the current study's measures from time points 1 and 3 (0 units, 0.05 meters/second, and 11 seconds for the SPPB, $4 \mathrm{MUP}$, and $400 \mathrm{MW}$, respectively) would not be considered meaningful ( $p>0.05$ for mean changes, Table 2 ). Although the linear regression models for the $30 \mathrm{sCS}$ and $400 \mathrm{MW}$ were significant, suggesting proportional bias, the variance explained for both models was small (29\%), and the regression tests were underpowered, limiting interpretation. Taken together, the functional performance data presented in the current study demonstrated trivial changes, not considered clinically meaningful, supporting their consistency as a whole in older women over 15 weeks.

The inter/intrarater analyses produced ICCs that were high to extremely high, supporting the use of video-recorded functional performance assessment in older adults. Our findings are in agreement with other studies in clinical populations that have investigated the reliability of assessing functional performance remotely using video technology versus face-to-face testing $[34,35]$. For example, Cabana et al. reported very high to excellent ICCs for interrater (0.95-0.99) and intrarater (0.96-0.99) reliability for the 6minute walk test and the Timed Up and Go test in a group of stable heart failure patients that were tested in-person as well as remotely through video recordings. Aside from a few technical issues in recording functional performance (e.g., the recording stopped before the termination of a test), and ensuring sufficient lighting, test-marker placement, recording-device placement, and video-storage space, capturing functional performance in videos with the tests used 
in the current study provided a valid method to measure function that was reproducible (independent of the rater) and highly comparable to live testing. Thus, if standard procedures are followed, it is possible that videos captured by practitioners or participants themselves could be analysed to evaluate and monitor functional performance in older adults, thereby reducing the need for in-person testing.

\section{Limitations}

The current study's participants represent high-functioning, community-dwelling older women (baseline SPPB median score $=12$ units), and interpretation of results might not apply to clinical populations or frail older women, which is a limitation. In addition, the change in the mean for the chair stand tests (5 CS and $30 \mathrm{sCS}$ ) does impact the interpretation of the stability of these tests over 15 weeks in healthy, older women. The increase in chair stand performance, while not clinically meaningful, does reduce the long-term reliability of this measure and suggests a potential learning effect.

\section{Conclusions}

Lower extremity muscle power and functional tests were found to be reliable in older, healthy women over 15 weeks, given a standardized testing environment and administration, minimal familiarization in study design, and multiple raters. These measures were stable over time and can be used to detect changes in response to interventions in older, healthy women. Future research should examine the longterm reliability of functional test performance in healthy, older men, as well as clinical populations.

\section{Data Availability}

The data used in the study are available from the corresponding author upon request via email.

\section{Disclosure}

The current manuscript has been presented at the American College of Sports Medicine Virtual Meeting 2020 in abstract form in a poster presentation. It has not been published nor is under review elsewhere.

\section{Conflicts of Interest}

The authors declare that there are no conflicts of interest regarding the publication of this article.

\section{Acknowledgments}

The authors would like to acknowledge Samantha Jeske, Rachel Goodman, and Cristiane Cruz for their contributions to the current study.

\section{Supplementary Materials}

The means (SD) for all participants (regardless of missing data) are shown in Tables S1 (torque and power) and S2 (functional performance). Bland-Altman plots for all measures using time points 1 and 3 are shown in Figures S1-S14. (Supplementary Materials)

\section{References}

[1] M. Inzitari, "Qualitative neurological gait abnormalities, cardiovascular risk factors and functional status in older community-dwellers without neurological diseases: the healthy brain project," Experimental Gerontology, vol. 124, Article ID 110652, 2019.

[2] G. Karege, "Gait speed is associated with death or readmission among patients surviving acute hypercapnic respiratory failure," BMJ Open Respiratory Research, vol. 7, no. 1, 2020.

[3] S. Studenski, "Gait speed and survival in older adults," JAMA, vol. 305, no. 1, pp. 50-58, 2011.

[4] K. F. Reid and R. A. Fielding, "Skeletal muscle power," Exercise and Sport Sciences Reviews, vol. 40, no. 1, pp. 4-12, 2012.

[5] M. E. Jacob, M. M. Marron, R. M. Boudreau, M. C. Odden, A. M. Arnold, and A. B. Newman, "Age, race, and gender factors in incident disability," The Journals of Gerontology: Series A, vol. 73, no. 2, pp. 194-197, 2018.

[6] R. K. Wong, "Reliability and validity of the acquired brain injury challenge assessment (ABI-CA) in children," Brain Injury, vol. 28, no. 13-14, pp. 1734-1743, 2014.

[7] R. W. Bohannon, "Test-retest reliability of measurements of hand-grip strength obtained by dynamometry from older adults: a systematic review of research in the pubmed database," The Journal of Frailty \& Aging, vol. 6, no. 2, pp. 83-87, 2017.

[8] A. Goldberg, M. Chavis, J. Watkins, and T. Wilson, "The fivetimes-sit-to-stand test: validity, reliability and detectable change in older females," Aging Clinical and Experimental Research, vol. 24, no. 4, pp. 339-344, 2012.

[9] S. Van Driessche, "Test-retest reliability of knee extensor rate of velocity and power development in older adults using the isotonic mode on a biodex system 3 dynamometer," PLoS One, vol. 13, no. 5, Article ID e0196838, 2018.

[10] B. Buehring, D. Krueger, E. Fidler, R. Gangnon, B. Heiderscheit, and N. Binkley, "Reproducibility of jumping mechanography and traditional measures of physical and muscle function in older adults," Osteoporosis International, vol. 26, no. 2, pp. 819-825, 2015.

[11] J. E. Morley, "Frailty and sarcopenia in elderly," Wien Klin Wochenschr, vol. 128, no. 7, pp. 439-445, 2016.

[12] C. Byrne, C. Faure, D. J. Keene, and S. E. Lamb, "Ageing, muscle power and physical function: a systematic review and implications for pragmatic training interventions," Sports Medicine, vol. 46, no. 9, pp. 1311-1332, 2016.

[13] J. Kottner, L. Audigé, S. Brorson et al., "Guidelines for reporting reliability and agreement studies (GRRAS) were proposed," Journal of Clinical Epidemiology, vol. 64, no. 1, pp. 96-106, 2011.

[14] S. D. Walter, M. Eliasziw, and A. Donner, "Sample size and optimal designs for reliability studies," Statistics in Medicine, vol. 17, no. 1, pp. 101-110, 1998.

[15] C. Hurst, A. M. Batterham, K. L. Weston, and M. Weston, "Short- and long-term reliability of leg extensor power measurement in middle-aged and older adults," Journal of Sports Sciences, vol. 36, no. 9, pp. 970-977, 2018.

[16] J. M. Guralnik, E. M. Simonsick, L. Ferrucci et al., "A short physical performance battery assessing lower extremity function: association with self-reported disability and 
prediction of mortality and nursing home admission," Journal of Gerontology, vol. 49, no. 2, pp. M85-M94, 1994.

[17] M. M. McDermott, K. Liu, J. M. Guralnik et al., "Functional decline in patients with and without peripheral arterial disease: predictive value of annual changes in levels of C-reactive protein and D-dimer," The Journals of Gerontology Series A: Biological Sciences and Medical Sciences, vol. 61, no. 4, pp. 374-379, 2006.

[18] C. J. Jones, R. E. Rikli, and W. C. Beam, "A 30-s chair-stand test as a measure of lower body strength in community-residing older adults," Research Quarterly for Exercise and Sport, vol. 70, no. 2, pp. 113-119, 1999.

[19] M. Ni, L. G. Brown, D. Lawler, and J. F. Bean, "Reliability, validity, and minimal detectable change of four-step stair climb power test in community-dwelling older adults," Physical Therapy, vol. 97, no. 7, pp. 767-773, 2017.

[20] E. M. Simonsick, P. S. Montgomery, A. B. Newman, D. C. Bauer, and T. Harris, "Measuring fitness in healthy older adults: the health ABC long distance corridor walk," Journal of the American Geriatrics Society, vol. 49, no. 11, pp. 1544-1548, 2001.

[21] A. Ghasemi and S. Zahediasl, "Normality tests for statistical analysis: a guide for non-statisticians," International Journal of Endocrinology and Metabolism, vol. 10, no. 2, pp. 486-489, 2012.

[22] S. C. Webber and M. M. Porter, "Reliability of ankle isometric, isotonic, and isokinetic strength and power testing in older women," Physical Therapy, vol. 90, no. 8, pp. 1165-1175, 2010.

[23] F. Faul, E. Erdfelder, A.-G. Lang, and A. Buchner, "G* power 3: a flexible statistical power analysis program for the social, behavioral, and biomedical sciences," Behavior Research Methods, vol. 39, no. 2, pp. 175-191, 2007.

[24] A. P. Field, Discovering Statistics Using IBM SPSS Statistics: And Sex and Drugs and Rock "N" Roll, Sage, Los Angeles, CA, USA, 4th edition, 2013.

[25] G. A. Power, B. H. Dalton, C. L. Rice, and A. A. Vandervoort, "Reproducibility of velocity-dependent power: before and after lengthening contractions," Applied Physiology, Nutrition, and Metabolism, vol. 36, no. 5, pp. 626-633, 2011.

[26] D. M. Mijnarends, J. M. M. Meijers, R. J. G. Halfens et al., "Validity and reliability of tools to measure muscle mass, strength, and physical performance in community-dwelling older people: a systematic review," Journal of the American Medical Directors Association, vol. 14, no. 3, pp. 170-178, 2013.

[27] G. V. Ostir, S. Volpato, L. P. Fried, P. Chaves, and J. M. Guralnik, "Reliability and sensitivity to change assessed for a summary measure of lower body function," Journal of Clinical Epidemiology, vol. 55, no. 9, pp. 916-921, 2002.

[28] M.-D. Morard, D. Besson, D. Laroche, A. Naaïm, V. Gremeaux, and J.-M. Casillas, "Fixed-distance walk tests at comfortable and fast speed: potential tools for the functional assessment of coronary patients?" Annals of Physical and Rehabilitation Medicine, vol. 60, no. 1, pp. 13-19, 2017.

[29] L. D. Westbury, H. E. Syddall, N. R. Fuggle et al., "Long-term rates of change in musculoskeletal aging and body composition: findings from the health, aging and body composition study," Calcified Tissue International, vol. 106, no. 6, pp. 616-624, 2020.

[30] G. A. Power, B. H. Dalton, C. L. Rice, and A. A. Vandervoort, "Power loss is greater following lengthening contractions in old versus young women," Age, vol. 34, no. 3, pp. 737-750, 2012.
[31] S. E. Jones, S. S. C. Kon, J. L. Canavan et al., "The five-repetition sit-to-stand test as a functional outcome measure in COPD," Thorax, vol. 68, no. 11, pp. 1015-1020, 2013.

[32] R. M. Alfonso-Rosa, B. del Pozo-Cruz, J. del Pozo-Cruz, B. Sañudo, and M. E. Rogers, "Test-retest reliability and minimal detectable change scores for fitness assessment in older adults with type 2 diabetes," Rehabilitation Nursing, vol. 39, no. 5, pp. 260-268, 2014.

[33] S. Kwon, S. Perera, M. Pahor et al., "What is a meaningful change in physical performance? Findings from a clinical trial in older adults (the Life-P study)," The Journal of Nutrition, Health and Aging, vol. 13, no. 6, pp. 538-544, 2009.

[34] F. Cabana, P. Boissy, M. Tousignant, H. Moffet, H. Corriveau, and R. Dumais, "Interrater agreement between telerehabilitation and face-to-face clinical outcome measurements for total knee arthroplasty," Telemedicine and E-Health, vol. 16, no. 3, pp. 293-298, 2010.

[35] R. Hwang, A. Mandrusiak, N. R. Morris, R. Peters, D. Korczyk, and T. Russell, "Assessing functional exercise capacity using telehealth: is it valid and reliable in patients with chronic heart failure?" Journal of Telemedicine and Telecare, vol. 23, no. 2, pp. 225-232, 2017. 